\title{
MECHANICAL MODEL FOR HUMAN BALANCING ON ROLLING BALANCE BOARD
}

\author{
Csenge A. Molnar ${ }^{a, b, *}, \operatorname{Ambrus~Zelei~}^{a}$, Tamas Insperger $^{a, b}$ \\ ${ }^{a}$ MTA-BME Lendület Human Balancing Research Group, Muegyetem Rakpart 5, Budapest, Hungary \\ ${ }^{b}$ Department of Applied Mechanics, Budapest University of Technology and Economics, Muegyetem Rakpart 5, \\ Budapest, Hungary \\ * corresponding author: csenge.molnar@mm.bme.hu
}

\begin{abstract}
A two-degree-of-freedom mechanical model was developed to analyze human balancing on rolling balance board in the frontal plane. The human nervous system is modeled as a proportionalderivative controller with constant feedback delay. The radius $R$ of the wheels and the board distance $h$ measured from the center of the wheel are adjustable parameters. Simulation results using the mechanical model were compared with real balancing trials recorded by an OptiTrack motion capture system. The goal of the paper is to investigate whether the two-degree-of-freedom model is accurate enough to model the balancing task and to introduce a stabilometry parameter in order to characterize balancing skill in case of different set of $R$ and $h$. The conclusion is that the angle of the upper body and the angle of the head also play an important role in the balancing process therefore a three- or four-degree-of-freedom model is more appropriate.
\end{abstract}

KEYWORDS: balance board, human balancing, stability, stabilizability, stabilometry, time delay.

\section{INTRODUCTION}

Nowadays, in the aging society, falls related to loss of balance are becoming the second most frequent reason for accidental death. Therefore the analysis and the understanding of human balancing and the development of tools to prevent falling are more and more important tasks in biomechanical researches.

From dynamics point of view, human balancing is the stabilization of the human body around an unstable equilibrium, namely, the upright posture. The stabilization process is controlled by the nervous system. Processing the signals perceived by the sensory organs, determining and realizing the necessary muscle movement to maintain the balance require certain amount of time, therefore the control concept applied by the human nervous system can be modeled as a delayed feedback mechanism, where the feedback delay corresponds to the reaction time. There are many concepts to describe the control mechanism of the human nervous system, such as proportional-derivative (PD) feedback [1 4], proportional-derivative-acceleration (PDA) feedback [5], clock- and event-driven intermittent control 6- 9, act-and-wait [10, drift-and-act control [11] and predictor feedback [2, 5, 12. Sensory dead zones and sampled feedback mechanism can also be involved into the model of human balancing to model the chaotic behavior of the balancing movements [13].

Models for human balancing involves many uncertain components. Besides the lack of knowledge of the exact feedback mechanism, the control gains and the value of the reaction time delay are all uncertain parameters that have to be identified by some fitting technique. The mechanical model of the human body also involves many uncertain parameters, i.e., the mass and the inertia of the body segments [14, and the passive stiffness and damping at the joints [6, 15]. When human subjects are balancing on a balance board, then the mechanical properties of the balance board can be determined with high accuracy, therefore its actual parameters can be introduced into the mechanical model. Moreover, the parameters of the balance board applied in our research can be changed, thus the balancing performance at different conditions can be analyzed.

Most of the mechanical models for standing still involve a one-degree-of-freedom model, which is an inverted pendulum in the sagittal plane associated with ankle strategy [11, 16-18 and a four-bar mechanism in the frontal plane [19-21]. Two-degree-of-freedom models also exist in the literature, e.g., the double inverted pendulum associated with the hip strategy 22]. Balancing on a balance board in the frontal plane can be described by a two-degree-of-freedom model, since the angular position of the balance board plays an important role in the dynamics in addition to the position of the four-bar mechanism model of the human body.

The goal of this paper is to determine whether the one-degree-of-freedom four-bar mechanism model of the human body in the frontal plane is sufficient for dynamic models of balancing on a rolling balance board. Stabilizability in terms of the critical delay is compared to the balancing performance using the enveloping ellipse of the phase portrait as a stabilometry parameter. 

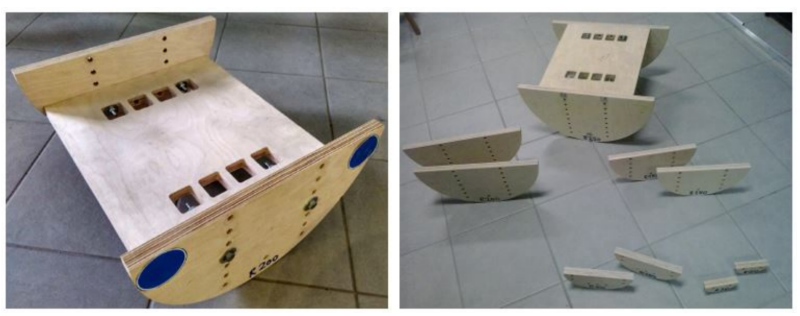

Figure 1. Balance board, with interchangeable wheels and adjustable board heights.

\section{BALANCE BOARD}

The balance board can be seen in Fig. 11 Two parameters can be changed: the radius $R$ of the wheels and the distance $h$ between the centre of the wheel and the board. The wheels were manufactured with radius of $50,750,100,125,150,200$ and $250 \mathrm{~mm}$ and parameter $h$ can be adjusted by steps of $25 \mathrm{~mm}$ in case of each wheel. Some of the wheels were manufactured with raised edge, which means, board distance $h$ can take negative value.

\section{BALANCING TASK}

The balancing task investigated in this paper is shown in Fig. 2. The balancing subjects have to balance on the balance board in the frontal plane, with stretched legs, open eyes and clasped hands behind the back. They have to press their foot to the board, the relative motion between the board and the feet can be prevented this way.

Changing the wheel radius or the board distance has an important role in terms of stabilizability of the system. Theoretical derivations and experimental results show that the smaller the wheel radius $R$ and the smaller the board distance $h$, the more difficult the balancing task 23 .

\section{MechanicAL MODEL}

A two-degree-of-freedom mechanical model was created for the balancing task, where the human body is modeled as a four-bar mechanism, as can be seen in Fig. 3. The legs are two homogeneous rigid bars and the trunk corresponds to a T-shaped rigid body connected to the legs by ideal pins. The feet are considered to be fixed to the board, and ankles behave as pins between the legs and the balance board. The generalized coordinates are the angle $\varphi$ of the trunk relative to the balance board and the angle $\vartheta$ of the balance board relative to the environment. The control concept of the human nervous system is described as a time-delayed PD controller with a constant time delay $\tau$, which corresponds to the reaction time of the balancing person. According to this assumption, the nervous system controls by signals proportional to the angular offset and velocity of the balance board and the human body. The control torque $M$ is assumed to act between the hip and the legs as indicated in

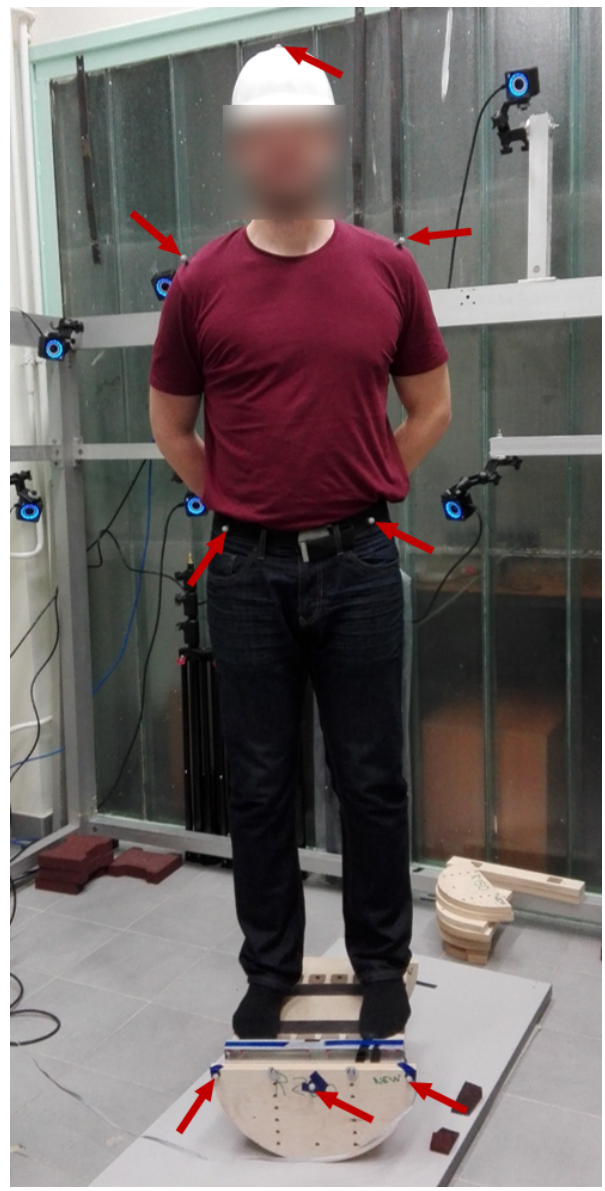

FiguRE 2. Balancing task, measurement setup.

Fig. 3. The passive stiffness $s_{a}$ and $s_{h}$ at the ankles and the hips is determined based on data from the literature [15, 24 27] assuming that both joints can be considered as spherical joints in case of small rotations.

Passive damping at the joints is neglected, therefore the equation of motion can be written as

$$
\mathbf{M} \ddot{\mathbf{q}}(t)+\mathbf{S q}(t)=\mathbf{P q}(t-\tau)+\mathbf{D} \dot{\mathbf{q}}(t-\tau),
$$

where $\mathbf{M}$ and $\mathbf{S}$ are the mass and the stiffness matrices,

$$
\mathbf{P}=\left[\begin{array}{cc}
P_{\varphi} & P_{\vartheta} \\
0 & 0
\end{array}\right], \quad \mathbf{D}=\left[\begin{array}{cc}
D_{\varphi} & D_{\vartheta} \\
0 & 0
\end{array}\right],
$$

are the matrices of the proportional and the derivative gains, respectively, and

$$
\mathbf{q}(t)=\left[\begin{array}{l}
\varphi(t) \\
\vartheta(t)
\end{array}\right]
$$

is the vector of generalized coordinates.

Mathematically, the equation (1) of motion is a system of linearized delayed differential equations. The stability analysis of the solution is performed using the semidiscretization method 28 . There exists a critical time delay $\tau_{\text {crit }}$ for each combination of $R$ and $h$, if the feedback delay is larger than $\tau_{\text {crit }}$ then there are no $P_{\varphi}, D_{\varphi}, P_{\vartheta}, D_{\vartheta}$ control gains associated with a 


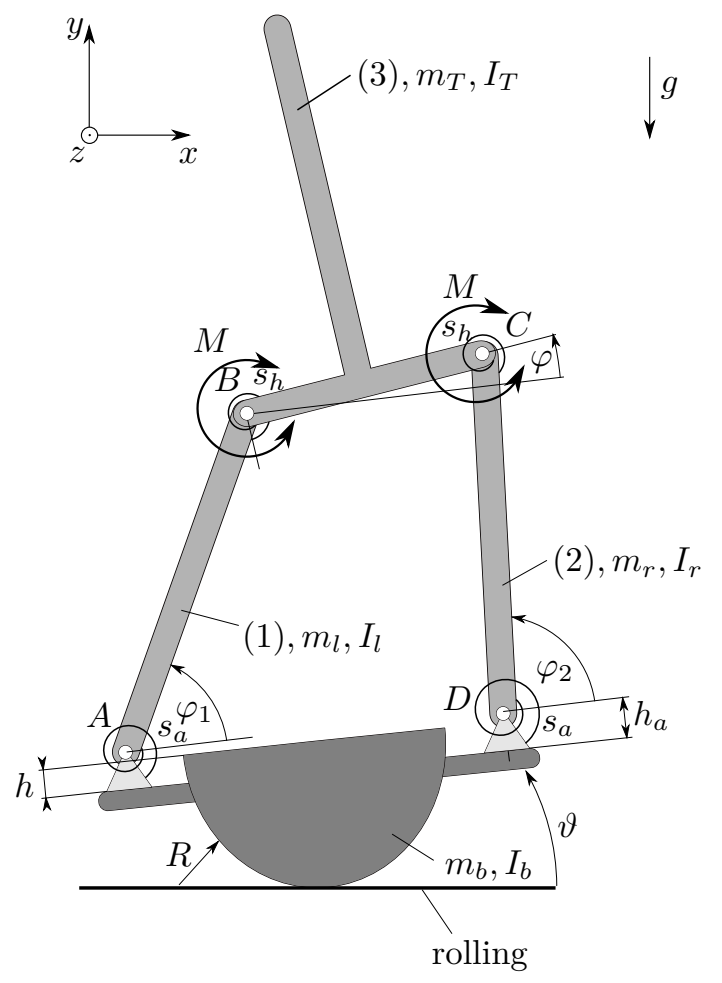

FiguRE 3. Two-degree-of-freedom mechanical model of balancing on a rolling balance board in the frontal plane.

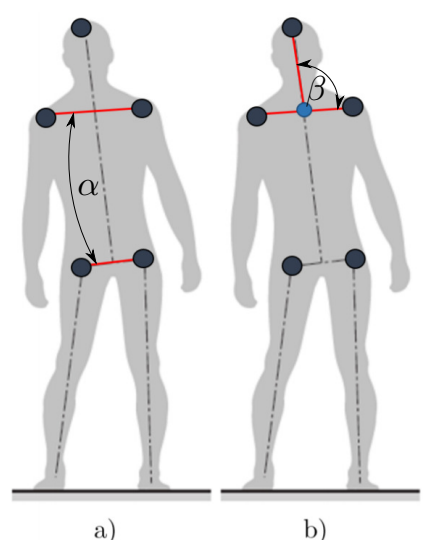

Figure 4. Definition of angles $\alpha$ and $\beta$.

stable system. A stabilizability diagram can be constructed for each balancing subject, which shows the critical time delay as function of the balance board geometry (see Fig. 8). Contour lines show, that the critical time delay decreases for smaller $R$ and $h$ values, which indicates that the balancing task is more difficult. Reference [23] contains a detailed description of the construction of the stabilizability diagram.

\section{Degrees of FREEDOM OF THE HUMAN BODY MODEL}

In order to validate the mechanical model, the balancing subject performed $60 \mathrm{~s}$ long balancing trials for a series of combinations of $R$ and $h$. The arrows in

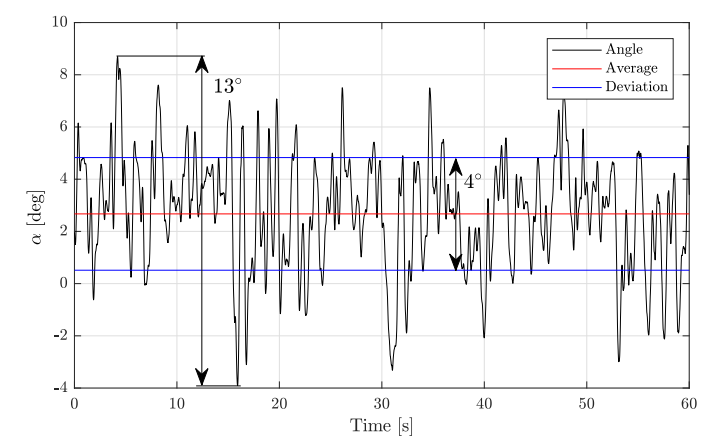

Figure 5. Time history of angle $\alpha$ indicated in Fig. 4 a).

Fig. 2 show the markers located on the human body and the balance board during the measurements. An OptiTrack motion capture system was used to record the $3 \mathrm{D}$ position of the markers. The sampling frequency was $120 \mathrm{~Hz}$. For a fixed wheel radius $R$, the halving method was used to determine the critical value of parameter $h$ where the subject was not able to balance at least for $60 \mathrm{~s}$.

According to the mechanical model, the whole upper body was modeled as one rigid body. In order to validate this assumption, two angles were specified to measure the change in the posture of the upper body. Angle $\alpha$ is the angle between the lines connecting the markers placed on the shoulders and on the hips (see Fig. 4a). Angle $\beta$ is the angle between the lines connecting the markers on the shoulders and the line connecting the midpoint of this line and the marker on the top of the head (see Fig. $4 \mathrm{~b}$ ). According to the rigid upper body assumption, angles $\alpha$ and $\beta$ should not change during the balancing trials.

The time history of angles $\alpha$ and $\beta$ can be seen in Fig. 5 and Fig. 6, respectively. The mean value and the standard deviation are indicated by red and blue lines, respectively. The difference between the highest and lowest value of the angles are also indicated. It can be seen that the motion of the shoulders relative to the hip is larger (standard deviation of $4^{\circ}$ and maximum difference of $13^{\circ}$ ) than the motion of the head with respect to the upper body (standard deviation of $3^{\circ}$ and maximum difference of $9^{\circ}$ ). Both variations are commensurable to the angles during the balancing trials (e.g., the angle of the legs with respect to the balance board). This observation implies that a more detailed multi-body model, which involves extra rigid bodies for the upper body and the head, is more appropriate. On the other hand, the introduction of extra rigid bodies in the model induces more uncertain parameters, such as the inertia of the upper body and the head, which may strongly affect the accuracy of the model. Therefore in the following analysis, we use the rigid upper body model. 


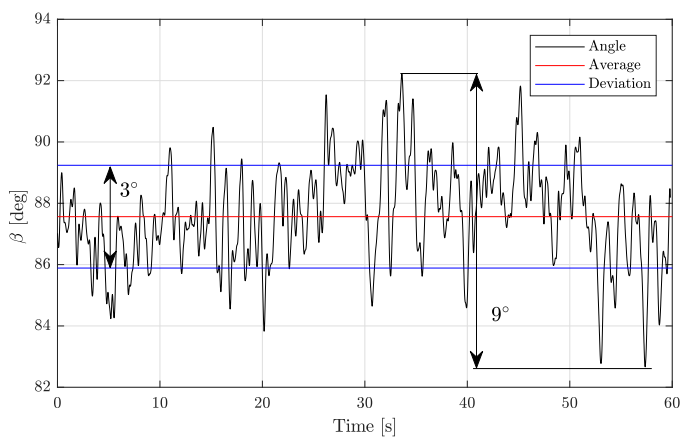

Figure 6. Time history of angle $\beta$ indicated in Fig. 4 b).

\section{Stabilometry}

Many different parameters can be found in the literature to characterize the performance during balancing tasks, such as mean power frequency, median frequency, frequency range power ratios, spectral power AP-ML ratio, largest amplitude and corresponding largest frequency, just to mention a few [29, 30. In a previous work of the authors [23, the standard deviation of the balance board angle was used as a measure of balancing skill. In this paper, a stabilometry parameter based on the phase portrait of the balance board angle is introduced since the magnitude of the angle and angular velocity of the balance board are both related to the performance during balancing. The angle of the balance board relative to the environment is calculated from the position of the markers located on the left and right corner of the wheel (Fig. 2) at each time step. The angular velocity is determined via discrete differentiation. An example for the corresponding phase portrait is shown in Fig. 7 together with an enveloping ellipse. The semi-major axis $a$ and $b$ of the ellipse is calculated as the half of the difference between the maximum and minimum angle and angular velocity of the balance board, respectively.

One can observe some parts on the phase portrait, where the density of the trajectories is lower, as shown by black in Fig. 7. These part of the trajectory are related to the loss of concentration of the balancing subject for a very short time and appear as larger peaks in the time history of the balance board angle. Before determining the ellipses, these regions caused by these temporary effect were eliminated. First, the normal probability density (NPD) of the balance board angle was calculated. Then, a threshold value of the NPD was determined and the values of the balance board angle below this threshold were eliminated. A similar process was used to filter the angular velocity. The semi-axes and the area of the ellipse were calculated using the filtered angle and angular velocity.

The result of the balancing performance by the subject can be seen in Fig. 8 Green circles refer to the $R$ and $h$ pairs, where the $60 \mathrm{~s}$ long balancing trial was

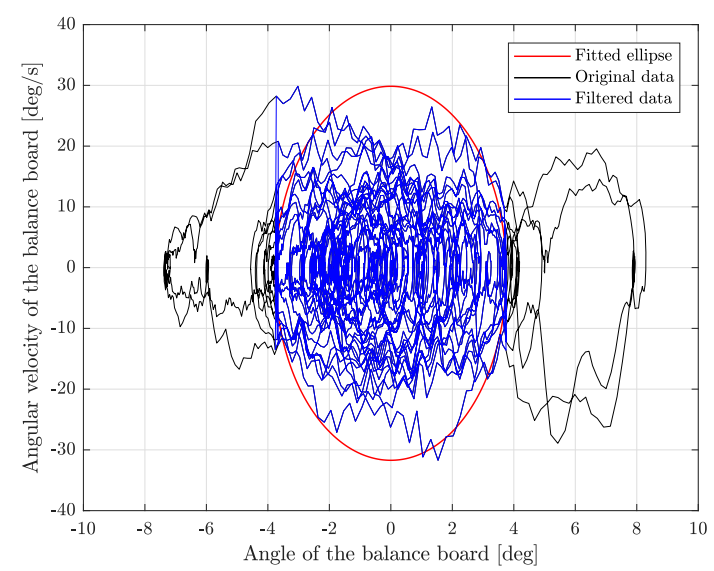

FIgURE 7. Phase portrait of the balance board angle, data filtering, and the fitted ellipse.

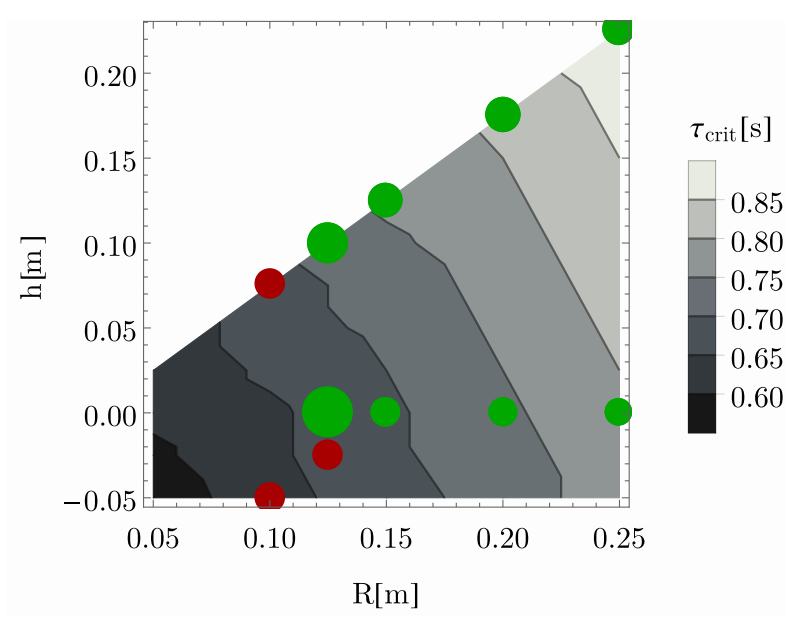

Figure 8. Stabilizability diagram.

successful. The diameter of the circle is proportional to the area of the fitted ellipse. Red circles indicates unstable trials. Grayscale indicates the critical delay determined using the mechanical model. One can observe, that the calculated stabilizability and the measurement has the same tendency. The balancing task is easier in case of larger radius and larger board distance, which meets the personal experience and opinion of the balancing subject.

\section{Conclusions}

Balancing performance by a human subject was compared to the critical reaction time delay obtained by the stabilizability analysis of the underlying mechanical model. Unsuccessful balancing trials allows an estimation of the reaction time delay of the balancing subject. The grey scale in Fig. 8 shows that if the reaction time is smaller than $0.85 \mathrm{~s}$, then the subject is able to balance on the lowest set of the balance board with wheel radius $R=250 \mathrm{~mm}$. Comparison 
of the value of the grey scale and the boundary between the successful and unsuccessful trials, which is approximately parallel to the contour lines of the stabilizability diagram, the reaction time is estimated to be $0.68 \mathrm{~s}$. According to the literature, the reaction time associated with human posture is definitely lower then this value. This difference can be explained by the simplified mechanical model, i.e., the upper body was modeled as a rigid body. A multi-body model, which involves additional rigid bodies for the upper body and for the head may be more appropriate. Another issue is that the feedback mechanism used in this model was a delayed PD control. In the literature, other types of control concepts are also often used to model human motor control. For instance, PDA control [5] or predictor feedback [2, 5, 12] are also possible candidates for the control law of human balancing tasks.

\section{ACKNOWLEDGEMENTS}

The research reported in this paper was supported by the ÚNKP-17-2-I New National Excellence Program of the Ministry of Human Capacities and by the Higher Education Excellence Program of the Ministry of Human Capacities in the frame of Biotechnology research area of Budapest University of Technology and Economics (BME FIKP-BIO).

\section{REFERENCES}

[1] J. R. Chagdes, S. Rietdyk, J. M. Haddad, et al. Limit cycle oscillations in standing human posture. Journal of biomechanics 49(7):1170-1179, 2016. DOI:10.1016/j.jbiomech.2016.03.005.

[2] B. Mehta, S. Schaal. Forward models in visuomotor control. Journal of Neurophysiology 88(2):942-953, 2002. DOI:10.1152/jn.2002.88.2.942

[3] P. Kowalcyzk, G. Glendinning, M. Brown, et al. Modeling stick balancing using switched systems with linear feedback control, jr soc. Interface 9:234-245, 2012.

[4] B. Varszegi, D. Takacs, G. Stepan, S. J. Hogan. Stabilizing skateboard speed-wobble with reflex delay. Journal of The Royal Society Interface 13(121):20160345, 2016. DOI:10.1098/rsif.2016.0345

[5] T. Insperger, J. Milton. Sensory uncertainty and stick balancing at the fingertip. Biological cybernetics 108(1):85-101, 2014. DOI:10.1007/s00422-013-0582-2

[6] Y. Asai, Y. Tasaka, K. Nomura, et al. A model of postural control in quiet standing: robust compensation of delay-induced instability using intermittent activation of feedback control. PLoS One 4(7):e6169, 2009. DOI:10.1371/journal.pone.0006169.

[7] J. L. Cabrera, J. G. Milton. On-off intermittency in a human balancing task. Physical Review Letters 89(15):158702, 2002. DOI:10.1103/PhysRevLett.89.158702

[8] N. Yoshikawa, Y. Suzuki, K. Kiyono, T. Nomura. Intermittent feedback-control strategy for stabilizing inverted pendulum on manually controlled cart as analogy to human stick balancing. Frontiers in computational neuroscience 10:34, 2016. DOI:10.3389/fncom.2016.00034.
[9] P. Gawthrop, I. Loram, H. Gollee, M. Lakie. Intermittent control models of human standing: similarities and differences. Biological cybernetics 108(2):159-168, 2014. DOI:10.1007/s00422-014-0587-5

[10] T. Insperger. Act-and-wait concept for continuous-time control systems with feedback delay. IEEE Transactions on Control Systems Technology 14(5):974-977, 2006. DOI:10.1109/TCST.2006.876938

[11] J. G. Milton, T. Ohira, J. L. Cabrera, et al. Balancing with vibration: a prelude for âĂIJdrift and actâĂİ balance control. PLoS One 4(10):e7427, 2009. DOI:10.1371/journal.pone.0007427

[12] J. Milton, R. Meyer, M. Zhvanetsky, et al. Control at stability's edge minimizes energetic costs: expert stick balancing. Journal of The Royal Society Interface 13(119):20160212, 2016. DOI:10.1098/rsif.2016.0212

[13] J. G. Milton, T. Insperger, W. Cook, et al. Microchaos in human postural balance: Sensory dead zones and sampled time-delayed feedback. Physical Review E 2018. In press.

[14] P. De Leva. Adjustments to Zatsiorsky-Seluyanov's segment inertia parameters. Journal of biomechanics 29(9):1223-1230, 1996. DOI:10.1016/0021-9290(95)00178-6

[15] I. D. Loram, M. Lakie. Direct measurement of human ankle stiffness during quiet standing: the intrinsic mechanical stiffness is insufficient for stability. The journal of physiology 545(3):1041-1053, 2002. DOI:10.1113/jphysiol.2002.025049.

[16] C. Maurer, R. J. Peterka. A new interpretation of spontaneous sway measures based on a simple model of human postural control. Journal of neurophysiology 93(1):189-200, 2005. DOI:10.1152/jn.00221.2004.

[17] G. Stepan. Delay effects in the human sensory system during balancing. Philosophical Transactions of the Royal Society of London A: Mathematical, Physical and Engineering Sciences 367(1891):1195-1212, 2009. DOI:10.1098/rsta.2008.0278.

[18] S. Hwang, P. Agada, T. Kiemel, J. J. Jeka. Identification of the unstable human postural control system. Frontiers in systems neuroscience 10:22, 2016. DOI:10.3389/fnsys.2016.00022

[19] A. D. Goodworth, R. J. Peterka. Influence of stance width on frontal plane postural dynamics and coordination in human balance control. Journal of Neurophysiology 104(2):1103-1118, 2010. DOI:10.1152/jn.00916.2009

[20] J. T. Bingham, J. T. Choi, L. H. Ting. Stability in a frontal plane model of balance requires coupled changes to postural configuration and neural feedback control. Journal of neurophysiology 106(1):437-448, 2011. DOI:10.1152/jn.00010.2011

[21] D. Hajdu, J. Milton, T. Insperger. Extension of stability radius to neuromechanical systems with structured real perturbations. IEEE transactions on neural systems and rehabilitation engineering 24(11):1235-1242, 2016. DOI:10.1109/TNSRE.2016.2541083 
[22] Y. Suzuki, T. Nomura, M. Casadio, P. Morasso. Intermittent control with ankle, hip, and mixed strategies during quiet standing: a theoretical proposal based on a double inverted pendulum model. Journal of Theoretical Biology 310:55-79, 2012. DOI:10.1016/j.jtbi.2012.06.019

[23] C. A. Molnar, A. Zelei, T. Insperger. Human balancing on rolling balance board in the frontal plane. In 14 th IFAC Workshop on Time Delay Systems, pp. 1-6. Budapest, Hungary, 2018.

[24] D. A. Winter, A. E. Patla, F. Prince, et al. Stiffness control of balance in quiet standing. Journal of neurophysiology 80(3):1211-1221, 1998. DOI:10.1152/jn.1998.80.3.1211

[25] Y.-S. Yoon, J. Mansour. The passive elastic moment at the hip. Journal of biomechanics 15(12):905-910, 1982. DOI:10.1016/0021-9290(82)90008-2.

[26] R. Riener, T. Edrich. Identification of passive elastic joint moments in the lower extremities. Journal of biomechanics 32(5):539-544, 1999.

DOI:10.1016/S0021-9290(99)00009-3
[27] A. Silder, B. Whittington, B. Heiderscheit, D. G. Thelen. Identification of passive elastic joint moment-angle relationships in the lower extremity. Journal of biomechanics 40(12):2628-2635, 2007. DOI:10.1016/j.jbiomech.2006.12.017.

[28] T. Insperger, G. Stépán. Semi-discretization for timedelay systems: stability and engineering applications, vol. 178. Springer Science \& Business Media, 2011.

[29] G. Nagymáté, Z. Orlovits, R. M. Kiss. Reliability analysis of a sensitive and independent stabilometry parameter set. PloS one 13(4):e0195995, 2018. DOI:10.1371/journal.pone.0195995

[30] G. Nagymáté, R. M. Kiss. Parameter reduction in the frequency analysis of center of pressure in stabilometry. Periodica Polytechnica Mechanical Engineering 60(4):238-246, 2016. DOI:10.3311/PPme.8999. 